\title{
Fundamental constants in time from the big-bang
}

\author{
Heikki Sipilä and Ari Lehto \\ Physics Foundations Society \\ Espoo, Finland
}

\begin{abstract}
Our understanding and theoretical interpretation of observations in astrophysics and cosmology depends on our knowledge of the fundamental constants and their possible dependence on time and space. Atomic spectroscopy and radio astronomy give important information on the validity and stability of the fundamental constants. The possible dependence of the fine structure constant alpha on time and spatial direction is an active topic of research.

Period doubling is a universal property of nonlinear dynamical systems, and the doubling is exact in principle. The value of the elementary charge squared can be calculated by the period doubling process from the Planck charge and thereby the value of alpha.

If 'old' and 'new' electrons are identical, then the Planck charge, i.e. a set of natural constants, has remained constant over time. In this article we show that the value of alpha calculated from the Planck charge is $0.007 \%$ smaller than the current accepted value of alpha.
\end{abstract}

Keywords: Fine structure constant, period doubling, alpha, natural constants

\section{Introduction}

The well-known period doubling (PD) process occurring in various nonlinear dynamical systems [1-8], can be related to the Coulomb energy of the elementary charge and thereby to the fine structure constant alpha assuming that the electron-positron pair creation process is nonlinear, and that the natural constants (i.e. the Planck scale) determine the outcome. The period doubling process produces accurate values for the electron's mass and magnetic moment, too [9].

Period doubling means frequency halving or subharmonic creation starting at the fundamental period which is defined by the Planck time $\left(1.35 \cdot 10^{-43} \mathrm{~s}\right)$ in this case. Period doubling is exact, in principle.

In this article the fine structure constant is numerically analysed using the simple period doubling formulas for three and four degrees of freedom in the Coulombic system.

The fine structure constant alpha is defined

$$
\alpha=\frac{\mu_{o} c}{2 h} e^{2}
$$

and the Planck charge $q_{P}$ squared

$$
q_{P}^{2}=\frac{4 \pi h}{\mu_{o} c}
$$

Combining (1) and (2) yields

$$
\alpha=2 \pi \frac{e^{2}}{q_{P}{ }^{2}}
$$


where $\mu_{o}$ is the vacuum permeability, $h$ the Planck constant, $c$ the speed of light and $e$ the elementary electric charge. The ratio of charges squared in (3) represents the ratio of Coulomb energies. According to the Planck relation $E=h f=h / p e r i o d$, and therefore $h$ is used instead of $h$-bar in (1) and (2).

When period doubles, energy halves. If the period doubling phenomenon takes place at the Planck scale, then $e^{2}$ should result from $q_{P}{ }^{2}$ after several period doublings (or Coulomb energy halvings).

If period $t_{n}$ results from a one-degree-of-freedom period doubling process at the Planck scale, then

$$
t_{n}=2^{n} t_{p}
$$

where $t_{p}$ is the Planck time (period) and $n$ the number of period doublings.

The number of period doublings can be calculated from (4) by normalizing $t_{n}$, i.e. $t_{n}$ is presented as ratio to the reference value $t_{P}$ :

$$
\frac{t_{n}}{t_{p}}=2^{n}
$$

A real 3-dimensional system can oscillate about $x, y$, and $z$-axes (roll, pitch, yaw). These are mutually orthogonal oscillations and are normally called system degrees of freedom. Period doubling can take place in every degree of freedom independently, i.e. with independent number of doublings.

For a system with three periods $t_{i}, t_{j}$ and $t_{k}$ the corresponding geometric period-space shape is a straight parallelepiped, whose volume is $V_{i j k}=t_{i} t_{j} t_{k}$. The volume doubles if any one period doubles.

Lehto [10] has shown that our method of observation gives a characteristic period $t_{c}$, which is the geometric mean of the three periods:

$$
t_{c}^{3}=t_{i} t_{j} t_{k}
$$

and

$$
t_{c}=\left(t_{i} t_{j} t_{k}\right)^{\frac{1}{3}}
$$

and so

$$
\frac{t_{c}}{t_{p}}=2^{n}=2^{\frac{i+j+k}{3}}=2^{\frac{N}{3}}
$$

where $N=i+j+k$ is the total number of period doublings. The exponent of 2 in (8) is an integer divided by the number of the degrees of freedom, i.e. the ratio is cube root of $2^{N}$.

It is also known that periods of the form

$$
t_{n}=2^{2^{n}} t_{o}
$$

are especially stable [11]. In (9) $t_{o}$ is the fundamental period of the system and $n$ the number of period doublings.

For four degrees of freedom (8) becomes

$$
\frac{t_{c}}{t_{p}}=2^{n}=2^{\frac{l+m+n+p}{4}}=2^{\frac{N}{4}}
$$




\section{Coulomb energy of the elementary charge and its relation to alpha}

Equation (3) shows that the fine structure constant equals the ratio of the Coulomb energies of the elementary charge and the Planck charge multiplied by $2 \pi$. The periods in (10) can be replaced by the charges squared, because energy is $h /$ period. One obtains

$$
\frac{e^{2}}{q_{P}{ }^{2}}=2^{n}=2^{-9.7499} \cong 2^{-9.75}=2^{-\frac{39}{4}}
$$

The perceived exponent $n$ is $-39 / 4$, which means that there are four degrees of freedom in the Coulombic system, and that the total number of period doublings (or energy halvings) is $N=39$. The stability condition (9) is fulfilled, because $N=39=l+m+n+p=2^{0}+2^{1}+2^{3}+2^{5}$.

The value of the elementary charge can be calculated from (11). One obtains $e=1.6021 \mathrm{As}$, which deviates from the accepted value of $e$ by $0.003 \%$.

Inserting (11) into (3) yields $\alpha=0.00729688$ and the inverse of alpha $\alpha^{-1}=137.04481$. Because the period doubling process is exact in principle, this value reflects the value of the Planck charge at the early epoch when the natural constants obtained their values.

\section{Experimental determination of alpha}

The status of the experimental determination of alpha is given in $[12,13]$. Figure 1 shows that the same natural constants lead to different values of alpha.

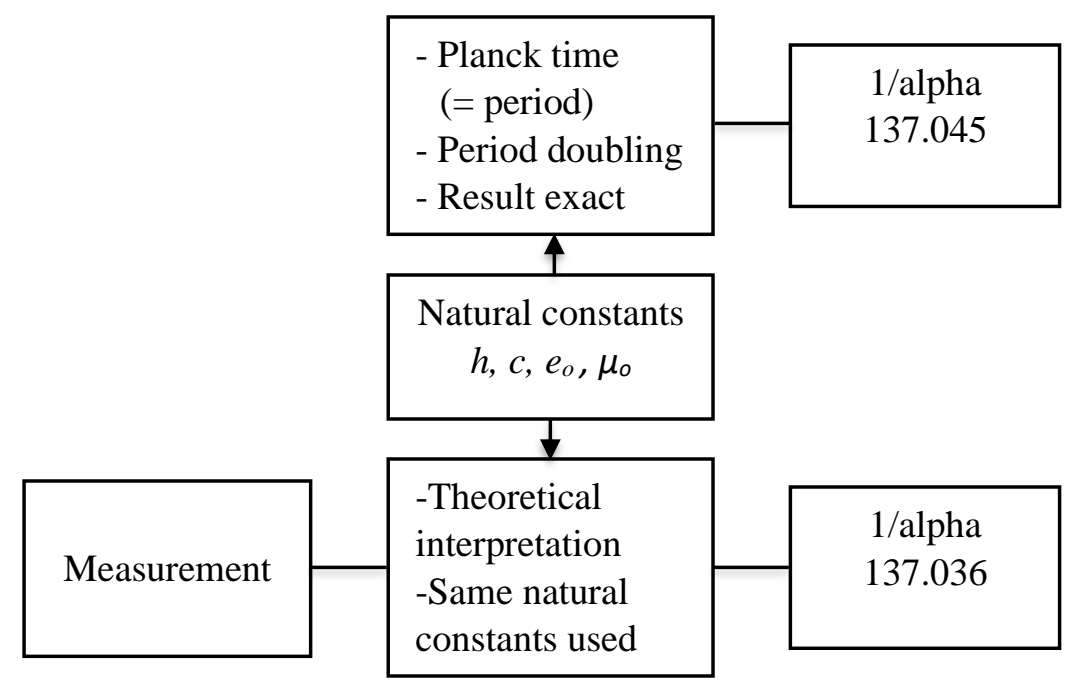

Fig. 1. The same set of natural constants leads to different values of alpha. In principle, period doubling is exact. The Planck charge is taken as the fundamental charge in the PD calculation.

It is generally assumed that all electrons are identical. This means that electrons born billions of years ago have the same physical properties as electrons born in a laboratory today. In view of the period doubling process the Planck charge, or the combination of the relevant natural constants, has remained constant over time.

The logical conclusion is that it is either the experimental setup or the theory used (or both) that have an influence on the value of alpha. For the objects outside the laboratory other explanations (in addition to the theory used) must be sought for. 


\section{Comparison}

Fine structure constant alpha is $\alpha=2 \pi \cdot 2^{-39 / 4}$ according to calculations based on periodic doubling. The NIST value for the inverse of alpha is 137.0359991, and the corresponding PD value is 137.04481 ( $0.007 \%$ difference). We propose that the periodic doubling value is more truthful because the doubling process faithfully follows the original (Planck) period.

We have not found any study which shows temporal change in the value of alpha. However, measurements from a quasar 13 billion years back in time show a relative change $(21.8 \pm$ 72.7) $\mathrm{ppm}$ of alpha over no variation model at the 3.9 sigma level [12]. There is no generally accepted theory of why alpha should have small spatial variations.

\section{Conclusions}

Period doubling is a well-known phenomenon in nonlinear dynamical systems. Here it is found that it accurately produces the value of the elementary charge squared and hence the value of the fine structure constant. If all electrons, 'old' and 'new', are identical, then this observation means that the combination of the fundamental constants defining the Planck charge has been exactly the same for billions of years.

\section{References}

[1] Faraday, M., 1831. On a peculiar class of acoustical figures and on certain forms assumed by groups of particles upon vibrating elastic surfaces, Philosophical Transactions of the Royal Society of London, 299-340.

[2] Rayleigh, L., Maintenance of vibrations by forces of double frequency, and propagation of waves through a medium with a periodic structure, Philosophical Magazine 24: 145-159 (1887).

[3] T. B. Simpson, J. M. Liu, A. Gavrielides, V. Kovanis, and P. M. Alsing, Period-doubling cascades and chaos in a semiconductor laser with optical injection, Phys. Rev. A 51, 4181, 1 May 1995. https://doi.org/10.1103/PhysRevA.51.4181

[4] R. Smolec, I. Soszy' nski, P. Moskalik, A. Udalski, M. K. Szyma' nski, M. Kubiak, G. Pietrzy'nski, Ł. Wyrzykowski, K. Ulaczyk, R. Poleski, S. Kozłowski and P. Pietrukowicz, Discovery of period doubling in BL Herculis stars of the OGLE survey. Observations and theoretical models, Mon. Not. R. Astron. Soc. 419, 2407-2423 (2012). https://doi.org/10.1111/j.1365-2966.2011.19891.x

[5] William G. Tifft, Redshift - Key to Cosmology, AlphaGraphics, ISBN 978-0-9862619-09,2014

[6] Young, D.L., Sheen, H.J. \& Hwu, T.Y. Period-doubling route to chaos for a swirling flow in an open cylindrical container with a rotating disk. Experiments in Fluids 18, 389-392 (1995). https://doi.org/10.1007/BF00211397

[7] Christopher J. Doona and Sory I. Doumbouya. Period-doubling route to chaos in the chlorite-thiocyanate chemical oscillator, J. Phys. Chem. 1994, 98, 2, 513-517, January 1, 1994, https://doi.org/10.1021/j100053a027

[8] Thomas Quail, Alvin Shrier, and Leon Glass, Predicting the onset of period-doubling bifurcations in noisy cardiac systems, PNAS|July 28, 2015|vol. 112|no. 30 9358-9363 www.pnas.org/cgi/doi/10.1073/pnas.1424320112 
[9] Lehto, A. Period Doubling Phenomenon as the Origin of Electron Properties. Preprints 2020, 2020060076 (doi: 10.20944/preprints202006.0076.v1).

[10] Ari Lehto: On the Planck Scale and Properties of Matter, International Journal of Astrophysics and Space Science. Special Issue: Quantum Vacuum, Fundamental Arena of the Universe: Models, Applications and Perspectives. Vol. 2, No. 6-1, 2015, pp. 57-65. https://doi.org/10.11648/j.ijass.s.2014020601.17

[11] M. J. Feigenbaum: Universal behavior in nonlinear systems, Physica 7D, (1983), 16-39

[12] Michael R.Wilczynska, John K.Webb, Matthew Bainbridge, John D.Barrow, Sarah E.I.Bosman, Robert E. Carswell, Mariusz P. Dabrowski, Vincent Dumont, Chung-Dhi Lee, Ana Catarina Leite, Katarzyna Leszczynska, Jochen Liske, Kondrad Marosek, Carlos J.A.P.Martins, Dinco Milakovic, Paolo Molaro, Luca Pasquini, Four direct measurements of the fine-structure constant 13 billion years ago, Sci.Adv..2020, 6

[13] Pierre Cladé, François Nez, François Biraben, Saïda Guellati-Khelifa, State of the art in the determination of the fine-structure constant and the ratio $h / m_{\mu}$. L'état de l'art de la détermination de la constante de structure fine et du rapport $h / m_{\mu}$, Comptes Rendus Physique Volume 20, Issues 1-2, January-February 2019, Pages 77-91, https://doi.org/10.1016/j.crhy.2018.12.003 